\title{
Flavor-Toxicant Correlation in E-cigarettes: A Meta-
}

\section{Analysis}

Sally Salam, BS, † Najat Aoun Saliba, PhD, †, Alan Shihadeh, ScD,,$\xi$ Thomas Eissenberg, $1, \S$ and Ahmad El-Hellani, PhD $\%$, , *

$\dagger$ Department of Chemistry, Faculty of Arts and Sciences, American University of Beirut, Beirut, Lebanon

¥ Department of Mechanical Engineering, Maroun Semaan Faculty of Engineering and Architecture, American University of Beirut, Beirut, Lebanon

1 Department of Psychology, Virginia Commonwealth University, Richmond, Virginia, USA

$\S$ Center for the Study of Tobacco Products, Virginia Commonwealth University, Richmond, Virginia, USA 
Table S1. A List of chemical compounds identified in the meta-analysis with structural information.

\begin{tabular}{|c|c|c|c|c|}
\hline Chemical & FG*1 & FG2 & FG3 & FG4 \\
\hline 1,4-Cineole & Ether & & & \\
\hline 1-Amylalcohol & Alcohol & & & \\
\hline 1-Ethenyloxy pentane & Alkene & Ether & & \\
\hline 1-Pentanol & Alcohol & & & \\
\hline 2,3,5,6-Tetramethylpyrazine & Pyrazine & & & \\
\hline 2,3,5-Trimethylpyrazine & Pyrazine & & & \\
\hline 2,3-Butanedione & Diketone & & & \\
\hline 2,3-Pentanedione & Diketone & & & \\
\hline 2,5-Dimethylpyrazine & Pyrazine & & & \\
\hline 2,6-Dimethyl-1,8-naphthyridine & Pyridine & & & \\
\hline 2,6-Dimethyl-4-propyl pyridine & Pyridine & & & \\
\hline 2,6-Dimethylpyridine & Pyridine & & & \\
\hline 2-Acetylpyridine & Ketone & Pyridine & & \\
\hline 2-Ethoxy-1-(3' pyridyl) ethylene & Alkene & Ether & Pyridine & \\
\hline 2-Isopropyl-4-methylthiazole & Thiazole & & & \\
\hline 2-Isopropyl-5-metylohex-2-enal & Aldehyde & Alkene & & \\
\hline 2-Methoxy-4-propylphenol & Ether & Phenol & & \\
\hline 2-Methylbutyl acetate & Ester & & & \\
\hline 2-Methylbutyrate & Ester & & & \\
\hline 2-Methylpyrazine & Pyrazine & & & \\
\hline 3-Methyl-1-phenyl-1H-pyrazole & Aryl & Pyrazole & & \\
\hline 3-Methylcoumarin & Alkene & Aryl & Ester & \\
\hline 3-Methylcyclopentane-1,2-dione & Diketone & & & \\
\hline 4-(4-Hydroxyphenyl)butan-2-one & Ketone & Phenol & & \\
\hline 4,4-Dimethyloxolan-2-one & Lactone & & & \\
\hline 4-Methoxy benzaldehyde & Aldehyde & Aryl & Ether & \\
\hline 4-Methylacetophenone & Aryl & Ketone & & \\
\hline 5-Methylfurfural & Aldehyde & Furan & & \\
\hline 6-Methyl-5-hepten-2-one & Alkene & Ketone & & \\
\hline 6-Methylcoumarin & Alkene & Aryl & Ester & \\
\hline 7-Methylcoumarin & Alkene & Aryl & Ester & \\
\hline Acetaldehyde hexamethylenehydrazone & Hydrazone & & & \\
\hline Acetoin & Alcohol & Ketone & & \\
\hline Acetophenone & Aryl & Ketone & & \\
\hline Acetylpyrazine & Ketone & Pyrazine & & \\
\hline Acetylpyrrole & Ketone & Pyrrole & & \\
\hline Allyl cyclohexanepropionate & Alkene & Ester & & \\
\hline Allyl hexanoate & Alkene & Ester & & \\
\hline Amyl acetate & Ester & & & \\
\hline
\end{tabular}


Amyl butyrate

Anethole

Anisyl acetate

Benzaldehyde

Benzyl acetate

Benzyl Alcohol

Benzyl Benzoate

Benzyl butyrate

Benzyl cinnamate

Benzyl propanoate

Butyl butyrate

Butyl butyrolactate

Caffeine

Camfor

Carvone

Cinnamaldehyde

Cinnamyl alcohol

Cinnamyl isovalerate

Cis-3-Hexenal

Cis-3-Hexenol

Cis-3-Hexenylacetate

Cis-3-Hexenylvalerate

Cis-Limonene oxide

Citral

Citronellol

Cocal

Corylone

Coumarin

Decanal

Diethyl succinate

Estragole

Ethanol

Ethyl 2-methyl butanoate

Ethyl 3-methyl-3-phenyloxirane-2-carboxylate

Ethyl acetate

Ethyl acetoacetate

Ethyl anthranilate

Ethyl butanoate

Ethyl caproate

Ethyl caprylate

Ethyl cinnamate

Ethyl heptanoate

Ethyl hexanoate
Ester

Alkene

Aryl Ether

Aryl

Ester Ether

Aldehyde

Aryl

Aryl

Ester

Alcohol

Aryl

Aryl

Ester

Aryl

Alkene

Ester

Aryl

Aryl

Ester

Ester

Ester

Pyrimidinedione Imidazole

Ketone

Ketone

Aldehyde

Alcohol

Alkene

Aldehyde

Alcohol

Alkene

Alkene

Alkene

Aldehyde

Alcohol

Aldehyde

Diketone

Alkene

Aldehyde

Ester

Alkene

Alcohol

Ester

Aryl

Ester

Ester

Aryl

Ester

Ester

Ester

Alkene

Ester

Ester
Epoxide Ester

Ketone

Ester

Amine

Alkene

Alkene Aryl

Alkene Aryl

Aryl Ester

Alkene

Alkene

Ester

Ester

Epoxide

Alkene

Alkene

Alkene Aryl

Aryl Lactone

Epoxide Ester

Aryl Ester

Aryl Ester
Aryl Ether 
Ethyl isovalerate

Ethyl lactate

Ethyl maltol

Ethyl phenylacetate

Ethyl propanoate

Ethyl Salicylate

Ethyl vanillin

Ethyl-methyl-hydroxymethyl amine

Eucalyptol

Eugenol

Fenchol

Furaneol

Furfural

Furfuryl alcohol

Geranyl propanoate

Guaiacol

Hedione

Heliotropine

Hemineurine

Hexanol

Hexyl 2-methylbutyrate

Hexyl acetate

Hexyl hexanoate

Hydrocoumarin

Hydroxyacetone

hydroxydihydrocarvone

Isoamyl butyrate

Isoamyl isovalerate

Isoamyl phenylacetate

Isobutyl acetate

Isopentyl acetate

Isopentyl alcohol

Isopulegol

Isosafroeugenol

Limonene

Linalool

Linalool oxide

Linalyl acetate

L-menthyl acetate

Luminol

Maltol

Melonal

Menthol
Ester

Alcohol

Alcohol

Aryl

Ester

Ester

Aldehyde

Alcohol

Ether

Alkene

Alcohol

Alcohol

Aldehyde

Alcohol

Alkene

Ether

Ester

Aldehyde

Thiazole

Alcohol

Ester

Ester

Ester

Aryl

Alcohol

Alcohol

Ester

Ester

Aryl

Ester

Ester

Alcohol

Alcohol

Alkene

Alkene

Alcohol

Alcohol

Alkene

Ester

Aryl

Alcohol

Aldehyde

Alcohol
Ester

Alkene Ether Ketone

Ester

Phenol

Ether Phenol

Amine

Ether Phenol

Alkene Ether Ketone

Furan

Furan

Ester

Phenol

Ketone

Aryl

Ether

Alcohol

Lactone

Ketone

Alkene Ketone

Ester

Alkene

Ether Phenol

Alkene

Alkene Ether

Ester

Amine Amide

Alkene Ether

Alkene

Ketone 
Menthone

Methyl 3-hydroxyhexanoate

Methyl anthranilate

Methyl cinnamate

Methyl cyclopentenolone

Methyl heptenone

Methyl salicylate

Neomenthol

Nerol

Nerol acetate

p-Anisalaldehyde

p-Cymene

Phenenthyl alcohol

Phenenthyl isovalerate

Piperitone

Piperonal

p-Tolualdehyde

Pulegone

Pyridine

Styralyl acetate

Tetrahydrolinalool

Theaspirane

Thymol

Trans-2-Hexenol

Triacetin

Vanillin

$\alpha, \alpha$-Dimethylphenethyl butyrate

$\alpha$-lonone

$\alpha$-Pinene

$\alpha$-Terpineol

$\beta$-Damascone

$\beta$-Ionone

$\beta$-Myrcene

$\beta$-Pinene

$\gamma$-Butyrolactone

$\gamma$-Decalactone

$\gamma$-Dodecalactone

$\gamma$-Hexalactone

$\gamma$-lonone

$\gamma$-Nonalactone

$\gamma$-Octalactone

$\gamma$-Terpinene

$\gamma$-Undecalactone
Ketone

Alcohol

Aryl

Alkene

Alcohol

Alkene

Ester

Alcohol

Alcohol

Alkene

Aldehyde

Aryl

Alcohol

Aryl

Alkene

Aldehyde

Aldehyde

Alkene

Pyridine

Aryl

Alcohol

Alkene

Phenol

Alcohol

Ester

Aldehyde

Aryl

Alkene

Alkene

Alcohol

Alkene

Alkene

Alkene

Alkene

Lactone

Lactone

Lactone

Lactone

Alkene

Lactone

Lactone

Alkene

Lactone
Ester

Ester Amine

Aryl Ester

Alkene Ketone

Ketone

Phenol

Alkene

Ester

Aryl Ether

Aryl

Ester

Ketone

Aryl

Aryl

Ketone

Ester

Ether

Alkene

Ether

Ester

Ketone

Alkene

Ketone

Ketone

Ketone

Ether 
$\mathrm{Y}$-Valerolactone

$\delta$-Decalactone

$\delta$-Dodecalactone

$\delta$-Tetradecalactone

$\delta$-undecalactone

Lactone

Lactone

Lactone

Lactone

Lactone

* FG: functional group. 